\title{
Flibanserin Versus Vardenafil in the Treatment of Female Sexual Dysfunction, Randomized Controlled Clinical Trial
}

Abdalla Kandil, Amany Nassar, Rasha Sayed Ahmed Mahmoud Serag

Department of Dermatology, Venereology and Andrology, Faculty of Medicine - Zagazig University. *Corresponding Author: Rasha Sayed Ahmed Mahmoud Serag, Mobile: (+20)01001541538, Email: rashaserag61@ gmail.com

\begin{abstract}
Background: Female sexual dysfunction (FSD) defined as the persistent/recurring decrease in sexual desire or arousal, the difficulty/inability to achieve an orgasm, and/or the feeling of pain during sexual intercourse. Therapeutic options available are few pharmacological options in the treatment of FSD.

Objective: To study the efficacy of flibanserin and vardenafil on female sexual dysfunction and identify which is the best. Methods: The study was conducted in Gynecology and Obstetrics Department and Dermatology and Venereology Department, Faculty of Medicine, Zagazig University Hospitals during the period from February 2019 to December 2019. Thirty-two married female patients were included in this study. These patients were divided into two groups. Group I included sixteen patients who were treated by vardenafil and group II included the sixteen patients who were treated by flibanserin.

Results: The present study showed that there were no statistical significant differences between the studied groups in any of demographic data. In addition, there were no statistical significant differences between the two studied groups in all scores pre-treatment. However, there was statistical significant increase in desire, orgasm and total score among Group II (flibanserin) compared to Group I (vardenafil) post-treatment.

Conclusion: Treatment of FSD is multi-factorial. Medications alone do not resolve FSD. Flibanserin is a controversial drug approved for a controversial disorder amid huge controversy. While it may serve as the light in the long search for female sexual problems, which still has a long way to go. Women taking this drug must well be educated about the adverse events associated with this drug and the possible interactions.
\end{abstract}

Keywords: Flibanserin, Vardenafil, Female Sexual Dysfunction.

\section{INTRODUCTION}

Female sexual dysfunction defined as the persistent/recurring decrease in sexual desire or arousal, the difficulty/inability to achieve an orgasm, and/or the feeling of pain during sexual intercourse ${ }^{(\mathbf{1})}$.

Therapeutic options available are few pharmacological options in the treatment of FSD. Historically, FSD patients were treated through psychological therapy. More basic science research and clinical recognition have been developed to address the problem. Several pharmacological initiatives are in development aimed at increasing blood flow to the genitals, improving androgen deficiencies and enhancing central nervous system stimulation ${ }^{(2)}$.

Flibanserin is a non-hormonal, centrally acting molecule that acts as an agonist at postsynaptic 5-HT1A receptors and as an antagonist at 5-HT2A receptor ${ }^{(3)}$. Flibanserin administration has been shown to lead to brain region-specific decreases in serotonin (5-HT) and increases in dopamine and norepinephrine ${ }^{(4)}$.

Vardenafil, in smooth muscle cells, nitric oxide activates the guanylate cyclase enzyme, which converts guanosine triphosphate into cyclic guanosine monophosphate. This molecule promotes the relaxation of the smooth muscle cells, causes vasodilatation, and increases blood flow in genital organs. The engorgement of clitoris and labia minora in women are the main modifications of genital organs during sexual arousal. The ultrafiltration of plasma through capillary vaginal vessels contributes to vaginal lubrication ${ }^{(5)}$.

Phosphodiesterase type 5 (PDE5) inhibitors (eg, sildenafil, tadalafil, vardenafil) physiologically enhance the production of guanosine monophosphate from cyclic guanosine monophosphate. PDE5 is expressed in vaginal, clitoral, and labial smooth muscles. Thus, PDE5 inhibitors could be used as an easily available medical treatment for genital FSADs ${ }^{\left({ }^{(}\right)}$.

The study aimed to study the efficacy of flibanserin and vardenafil on female sexual dysfunction and identify which is the best.

\section{PATIENTS AND METHODS}

The study was conducted in Gynecology and Obstetrics Department and Dermatology and Venereology Department, Faculty of Medicine, Zagazig University Hospitals during the period from Feb 2019 to December 2019.

\section{1) Sample size:}

Thirty two married female patients were included in this study. These patients were divided into two groups. Group 1 included sixteen patients who were treated by vardenafil and group II, which included sixteen patients who were treated by flibanserin. 


\section{Ethical approval:}

The protocol was approved by Scientific and Ethical committees, Faculty of Medicine, Zagazig University. A signed written consent was obtained from each patient.

Inclusion criteria: Married female patients complaining of sexual dysfunction more than 6 months with sexually active partner.

\section{Exclusion criteria:}

- Patients with sexual dysfunction caused by localized disorders.

- Patients cannot be treated with PDEs inhibitors type 5 (vardenafil):

1) Major hematological, renal or hepatic abnormalities.

2) Patients with major psychological disorders including major depression or psychosis.

3) A history of stroke or myocardial infarction or a significant cardiovascular disease within the last 6 months.

4) Concomitant treatment with nitrate.

- Patients cannot be treated with flibanserin

1) Renal failure

2) Hepatic failure

3) Pregnancy and lactation

- Patients with retinal problem.

- Patients with chronic debilitating diseases or hormonal disturbance.

- Psychatic distress especially depression.

\section{2) Operational design:}

Type of study:

A clinical trial and all included patients will be classified into two groups:

- Group I: 16 patients with FSD received vardenafil tablets $10 \mathrm{mg}$ at bedtime as oral dose for 2 months.

- Group II: 16 patients with FSD received $100 \mathrm{mg}$ flibanserin at bedtime as oral dose for 2 months.

\section{METHODS AND OBJECTIVES}

All patients in the $\mathbf{2}$ groups were subjected to:

1) Complete history taking: age, education, occupation, residence, age of marriage, special habits, history of medical diseases, surgical history and sexual history in the previous 6 months.

2) General and physical examination: pulse, blood pressure, routine laboratory investigations including CBC, LFT, RFT, RBS and lipid profiles.

3) Evaluation questionnaire: The questionnaire used included 25 items designed by the investigators (7). Only some items were selected from the female sexual function index (FSFI). Other questions were added to suit the purpose of study.
The FSFI, a 19-item questionnaire, has been developed as a brief, multidimensional self-report instrument for assessing the key dimensions of sexual function in women including six domains (desire, arousal, lubrication, orgasm, satisfaction and pain) as shown in table (1). It is psychometrically sound, easy to administer, and has demonstrated ability to discriminate between clinical and nonclinical populations. The questionnaire described was designed and validated for assessment of female sexual function and quality of life in clinical trials or epidemiological studies. Its further use in these areas remains to be investigated.

Table (1): Domain of sexual functions questionnaire (SFQ).

\begin{tabular}{|c|c|c|c|c|c|}
\hline Domain & Questions & \begin{tabular}{|l} 
Score \\
Range
\end{tabular} & Factor & $\begin{array}{l}\text { Min } \\
\text { Score }\end{array}$ & \begin{tabular}{|l} 
Max \\
Score
\end{tabular} \\
\hline Desire & 1,2 & $1-5$ & 0.6 & 1.2 & 6.0 \\
\hline Arousal & $3,4,5,6$ & $0-5$ & 0.3 & 0 & 6.0 \\
\hline Lubrication & $7,8,9,10$ & $0-5$ & 0.3 & 0 & 6.0 \\
\hline Orgasm & $11,12,13$ & $0-5$ & 0.4 & 0 & 6.0 \\
\hline Satisfaction & $14,15,16$ & \begin{tabular}{|c}
0 \\
(or1)- \\
5
\end{tabular} & 0.4 & 0.8 & 6.0 \\
\hline Pain & $17,18,19$ & $0-5$ & 0.4 & 0 & 6.0 \\
\hline \multicolumn{4}{|c|}{ Full Score Range } & 2.0 & 36.0 \\
\hline
\end{tabular}

4) Depression questionnaire to exclude the major psychological depressive disorder and its result put with the evaluation questionnaire as one item only.

\section{Statistical analysis:}

The collected data were computerized and statistically analyzed using SPSS program (Statistical Package for Social Science) version 18.0. Qualitative data were represented as frequencies and relative percentages. Chi square test was used to calculate difference between qualitative variables. Mann Whiteny (MW) test was used to calculate difference between quantitative variables in 2 groups in not normally distributed data. Paired t test was used to calculate difference between quantitative variables in the same group in 2 different times in normally distributed data. Paired Wilixocon test was used to calculate difference between quantitative variables in the same group in 2 different times in not normally distributed data. The threshold of significance is fixed at $5 \%$ level (P-value): $\mathrm{P}$ value of $>0.05$ indicates nonsignificant results. $\mathrm{P}$ value of $\leq 0.05$ indicates significant results.

\section{RESULTS}

There were no statistical significant differences between the studied groups in any of demographic data (Table 2). Table (3) showed that there were highly statistically significant increase in scores of lubrication and satisfaction $(37.5 \%$ \& $42.7 \%$ respectively) posttreatment. In addition, there was 
statistical significant increase in orgasm and total score ( $25 \% \& 23.36 \%$ respectively) posttreatment compared to pre-treatment. Table (4) showed that there was highly statistically significant increase in desire, orgasm, satisfaction and total $(33.3 \%, 41.76 \% \& 25.66$ $\%$ respectively) posttreatment. Moreover, there was statistical significant increase in arousal score $(23.75 \%$, $\%)$ posttreatment compared to pretreatment.
Table (5) showed that there were no statistical significant differences between the two studied groups in all scores pretreatment.

Table (6) showed that there was statistical significant increase in desire, orgasm and total score among group II compared to group I posttreatment.

Table (2): Comparison of demographic data of the two studied groups.

\begin{tabular}{|c|c|c|c|c|c|c|c|c|c|}
\hline & \multirow{4}{*}{ Variable } & \multirow{3}{*}{\multicolumn{2}{|c|}{$\begin{array}{l}\text { Total } \\
(n=32)\end{array}$}} & \multirow{3}{*}{\multicolumn{2}{|c|}{$\begin{array}{c}\text { Group I } \\
\text { (Vardenafil) } \\
(n=16) \\
\end{array}$}} & \multirow{3}{*}{\multicolumn{2}{|c|}{$\begin{array}{c}\text { Group II } \\
\text { (Flibanserin) } \\
(n=16) \\
\end{array}$}} & \multirow{4}{*}{$\chi^{2}$} & \multirow{4}{*}{$\mathbf{P}$} \\
\hline & & & & & & & & & \\
\hline & & & & & & & & & \\
\hline & & No & $\%$ & No & $\%$ & No & $\%$ & & \\
\hline \multirow{3}{*}{ Age: (years) } & $<20$ & 2 & 6.2 & 1 & 6.3 & 1 & 6.3 & \multirow{3}{*}{0.16} & \multirow{2}{*}{0.92} \\
\hline & $20-29$ & 21 & 65.6 & 10 & 62.5 & 11 & 68.7 & & \\
\hline & $\geq 30$ & 9 & 28.1 & 5 & 31.3 & 4 & 25 & & NS \\
\hline \multirow{3}{*}{ Education: } & Read \& write & 0 & 0 & 0 & 0 & 0 & 0 & \multirow{3}{*}{3.14} & \multirow[b]{2}{*}{0.08} \\
\hline & $\begin{array}{l}\text { Basic } \\
\text { education }\end{array}$ & 15 & 46.9 & 10 & 62.5 & 5 & 31.2 & & \\
\hline & University & 17 & 53.1 & 6 & 37.5 & 11 & 68.8 & & NS \\
\hline \multirow{2}{*}{ Occupation: } & Yes & 11 & 34.4 & 8 & 50 & 3 & 18.8 & \multirow{2}{*}{3.46} & 0.06 \\
\hline & No & 21 & 65.6 & 8 & 50 & 13 & 81.2 & & NS \\
\hline \multirow{2}{*}{ Residence: } & Urban & 16 & $50 \%$ & 5 & 31.3 & 11 & 68.7 & \multirow{2}{*}{3.8} & 0.15 \\
\hline & Rural & 16 & $50 \%$ & 11 & 68.7 & 5 & 31.2 & & NS \\
\hline \multirow{3}{*}{$\begin{array}{c}\text { Age of } \\
\text { marriage } \\
\text { (years): }\end{array}$} & $<20$ & 9 & 28.1 & 5 & 31.3 & 4 & 25 & \multirow{3}{*}{2.54} & \multirow{2}{*}{0.28} \\
\hline & $20-29$ & 21 & 65.6 & 9 & 56.2 & 12 & 75 & & \\
\hline & $\geq 30$ & 2 & 6.2 & 2 & 12.5 & 0 & 0 & & NS \\
\hline \multirow{3}{*}{$\begin{array}{l}\text { Husband's } \\
\text { age of } \\
\text { marriage } \\
\text { (years): }\end{array}$} & $<20$ & 0 & 0 & 0 & 0 & 0 & 0 & \multirow{3}{*}{0.53} & \multirow{2}{*}{0.47} \\
\hline & $20-29$ & 20 & 62.5 & 9 & 56.3 & 11 & 68.8 & & \\
\hline & $\geq 30$ & 12 & 37.5 & 7 & 43.8 & 5 & 31.2 & & NS \\
\hline
\end{tabular}

$\chi^{2}$ : Chi square test. NS: non-significant $(\mathrm{P}>0.05)$

Table (3): Comparison of sexual function index score before and after treatment in group I.

\begin{tabular}{|c|c|c|c|c|c|}
\hline \multicolumn{2}{|c|}{ Variable (vardenafil group): } & $\begin{array}{c}\text { Pre } \\
(n=16)\end{array}$ & $\begin{array}{c}\text { Post } \\
(n=16)\end{array}$ & $\mathbf{P}$ & $\%$ of \\
\hline Desire & $\begin{array}{c}\text { Mean } \pm \text { Sd } \\
\text { Median (Range) }\end{array}$ & $\begin{array}{c}4.88 \pm 1.82 \\
7(2-9)\end{array}$ & $\begin{array}{c}5.44 \pm 1.55 \\
6.5(3-9)\end{array}$ & $\begin{array}{l}0.72^{\wedge} \\
\mathrm{NS}\end{array}$ & $1.54 \%$ \\
\hline Arousal & $\begin{array}{c}\text { Mean } \pm \text { Sd } \\
\text { Median (Range) }\end{array}$ & $\begin{array}{c}2.25 \pm 0.58 \\
3(2-4)\end{array}$ & $\begin{array}{c}2.44 \pm 0.73 \\
3(2-4)\end{array}$ & $\begin{array}{l}0.19^{\wedge} \\
\mathrm{NS}\end{array}$ & $9.38 \%$ \\
\hline Lubrication & $\begin{array}{c}\text { Mean } \pm \text { Sd } \\
\text { Median (Range) }\end{array}$ & $\begin{array}{c}2.38 \pm 0.62 \\
3(2-4)\end{array}$ & $\begin{array}{c}3.19 \pm 0.54 \\
2(2-4)\end{array}$ & $\begin{array}{c}<0.001^{\wedge} \\
* *\end{array}$ & $37.5 \%$ \\
\hline Orgasm & $\begin{array}{c}\text { Mean } \pm \text { Sd } \\
\text { Median (Range) }\end{array}$ & $\begin{array}{c}1.63 \pm 0.62 \\
3(1-3)\end{array}$ & $\begin{array}{c}2.06 \pm 0.77 \\
3(1-3)\end{array}$ & $\begin{array}{c}\mathbf{0 . 0 2} \# \\
*\end{array}$ & $25 \%$ \\
\hline Satisfaction & $\begin{array}{c}\text { Mean } \pm \text { Sd } \\
\text { Median (Range) }\end{array}$ & $\begin{array}{c}2.5 \pm 0.63 \\
4(2-4)\end{array}$ & $\begin{array}{c}3.44 \pm 0.89 \\
3(2-5)\end{array}$ & $\begin{array}{c}\mathbf{0 . 0 0 1} \\
* *\end{array}$ & $42.71 \%$ \\
\hline Pain & $\begin{array}{c}\text { Mean } \pm \text { Sd } \\
\text { Median (Range) }\end{array}$ & $\begin{array}{c}0.5 \pm 0.5 \\
0.5(0-1)\end{array}$ & $\begin{array}{l}0.5 \pm 0.52 \\
0.5(0-1)\end{array}$ & $\begin{array}{l}1 \# \\
\text { NS }\end{array}$ & $0 \%$ \\
\hline Full score & $\begin{array}{c}\text { Mean } \pm \text { Sd } \\
\text { Median (Range) }\end{array}$ & $\begin{array}{l}14.13 \pm 2.73 \\
21(10-20) \\
\end{array}$ & $\begin{array}{r}17.1 \pm 2.46 \\
17(13-21) \\
\end{array}$ & $\begin{array}{c}\mathbf{0 . 0 0 1}_{* *}^{\wedge} \\
\end{array}$ & $23.36 \%$ \\
\hline
\end{tabular}

$\wedge$ : Paired t test \#: Paired Wilcoxon test

NS: Non significant $(\mathrm{p}>0.05) *$ : Significant $(\mathrm{P}<0.05) \quad * *$ : Highly significant $(\mathrm{p}<0.01)$

Table (4): Comparison of female sexual function index score before and after treatment in group II (flibanserin group).

\begin{tabular}{|c|c|c|c|c|c|}
\hline \multicolumn{2}{|c|}{ Variable } & Pre $(\mathbf{n}=\mathbf{1 6})$ & Post $(\mathbf{n}=\mathbf{1 6})$ & $\mathbf{P}$ & \% of \\
\hline Desire & Mean \pm Sd & $4.69 \pm 1.89$ & $6.45 \pm 1.17$ & $<\mathbf{0 . 0 0 1} \wedge$ & $\mathbf{3 3 . 3 \%}$ \\
\hline
\end{tabular}




\begin{tabular}{|c|c|c|c|c|c|}
\hline & Median (Range) & $8(2-8)$ & $5.5(4-10)$ & ** & \\
\hline Arousal & $\begin{array}{l}\text { Mean } \pm \text { Sd } \\
\text { Median (Range) }\end{array}$ & $\begin{array}{c}2.31 \pm 0.87 \\
3(1-4)\end{array}$ & $\begin{array}{c}2.88 \pm 0.89 \\
2(2-4)\end{array}$ & $\begin{array}{c}0.041 \# \\
*\end{array}$ & $23.75 \%$ \\
\hline Lubrication & $\begin{array}{l}\text { Mean } \pm \text { Sd } \\
\text { Median (Range) }\end{array}$ & $\begin{array}{c}2.63 \pm 1.09 \\
3(1-4)\end{array}$ & $\begin{array}{l}3.19 \pm 0.91 \\
1.5(2-4)\end{array}$ & $\begin{array}{c}0.66 \# \\
\text { NS }\end{array}$ & $26.88 \%$ \\
\hline Orgasm & $\begin{array}{l}\text { Mean } \pm \text { Sd } \\
\text { Median (Range) }\end{array}$ & $\begin{array}{c}1.75 \pm 0.86 \\
3(1-4)\end{array}$ & $\begin{array}{c}2.69 \pm 0.79 \\
2.5(2-4)\end{array}$ & $\begin{array}{c}\mathbf{0 . 0 0 4} \# \\
* *\end{array}$ & $38.13 \%$ \\
\hline Satisfaction & $\begin{array}{l}\text { Mean } \pm \text { Sd } \\
\text { Median (Range) }\end{array}$ & $\begin{array}{c}2.56 \pm 0.73 \\
4(2-4)\end{array}$ & $\begin{array}{c}3.63 \pm 0.72 \\
2(2-5)\end{array}$ & $\begin{array}{c}<0.001 \\
* *\end{array}$ & $41.67 \%$ \\
\hline Pain & $\begin{array}{l}\text { Mean } \pm \text { Sd } \\
\text { Median (Range) }\end{array}$ & $\begin{array}{c}0.56 \pm 0.51 \\
0(0-1)\end{array}$ & $\begin{array}{c}0.56 \pm 0.51 \\
0(0-1)\end{array}$ & $\begin{array}{l}1 \# \\
\text { NS }\end{array}$ & $0 \%$ \\
\hline Full score & $\begin{array}{l}\text { Mean } \pm \text { Sd } \\
\text { Median (Range) }\end{array}$ & $\begin{array}{c}14.5 \pm 3.25 \\
20(10-20)\end{array}$ & $\begin{array}{c}19.37 \pm 3.81 \\
14.5(13-25)\end{array}$ & $\begin{array}{c}<0.001 \\
* *\end{array}$ & $25.66 \%$ \\
\hline
\end{tabular}

Sd: Standard deviation.

NS: Non significant $(\mathrm{p}>0.05)$ $\wedge$ : Paired $t$ test

*: Significant $(\mathrm{P}<0.05)$
\#: Paired Wilcoxon test

**: Highly significant $(\mathrm{p}<0.01)$

Table (5): Comparison between the two studied groups in female sexual dysfunction index score before treatment.

\begin{tabular}{|c|c|c|c|c|c|}
\hline \multicolumn{2}{|c|}{ Variable } & $\begin{array}{c}\text { Group I (Vardenafil) } \\
(\mathbf{n}=16)\end{array}$ & $\begin{array}{c}\text { Group II (Flibanserin) } \\
(\mathbf{n}=16)\end{array}$ & Test & $\mathbf{P}$ \\
\hline Desire & $\begin{array}{c}\text { Mean } \pm \text { Sd } \\
\text { Median (Range) }\end{array}$ & $\begin{array}{l}4.88 \pm 1.82 \\
7(2-9)\end{array}$ & $\begin{array}{l}4.69 \pm 1.89 \\
8(2-8)\end{array}$ & $\begin{array}{c}\mathrm{t} \\
1.76\end{array}$ & $0.09 \mathrm{NS}$ \\
\hline Arousal & $\begin{array}{c}\text { Mean } \pm \text { Sd } \\
\text { Median (Range) }\end{array}$ & $\begin{array}{l}2.25 \pm 0.58 \\
3(2-4)\end{array}$ & $\begin{array}{l}2.31 \pm 0.87 \\
3(1-4)\end{array}$ & $\begin{array}{l}\text { MW } \\
-.16\end{array}$ & $0.87 \mathrm{NS}$ \\
\hline Lubrication & $\begin{array}{c}\text { Mean } \pm \text { Sd } \\
\text { Median (Range) }\end{array}$ & $\begin{array}{c}2.38 \pm 0.62 \\
3(2-4)\end{array}$ & $\begin{array}{c}2.63 \pm 1.09 \\
3(1-4)\end{array}$ & $\begin{array}{l}\text { MW } \\
-.59\end{array}$ & $0.56 \mathrm{NS}$ \\
\hline Orgasm & $\begin{array}{c}\text { Mean } \pm \text { Sd } \\
\text { Median (Range) }\end{array}$ & $\begin{array}{l}1.63 \pm 0.62 \\
3(1-3)\end{array}$ & $\begin{array}{l}1.75 \pm 0.86 \\
3(1-4)\end{array}$ & $\begin{array}{l}\text { MW } \\
-.21\end{array}$ & $0.84 \mathrm{NS}$ \\
\hline Satisfaction & $\begin{array}{c}\text { Mean } \pm \text { Sd } \\
\text { Median (Range) }\end{array}$ & $\begin{array}{l}2.5 \pm 0.63 \\
4(2-4)\end{array}$ & $\begin{array}{l}2.56 \pm 0.73 \\
4(2-4)\end{array}$ & $\begin{array}{c}\mathrm{t} \\
0.26\end{array}$ & $0.80 \mathrm{NS}$ \\
\hline Pain & $\begin{array}{c}\text { Mean } \pm \text { Sd } \\
\text { Median (Range) }\end{array}$ & $\begin{array}{l}0.5 \pm 0.5 \\
0.5(0-1)\end{array}$ & $\begin{array}{c}0.56 \pm 0.51 \\
0(0-1)\end{array}$ & $\begin{array}{l}\text { MW } \\
-.35\end{array}$ & $0.73 \mathrm{NS}$ \\
\hline Full score & $\begin{array}{c}\text { Mean } \pm \text { Sd } \\
\text { Median (Range) }\end{array}$ & $\begin{array}{l}14.13 \pm 2.73 \\
21(10-20)\end{array}$ & $\begin{array}{c}14.5 \pm 3.25 \\
20(10-20)\end{array}$ & $\begin{array}{c}\mathrm{t} \\
.35\end{array}$ & $0.73 \mathrm{NS}$ \\
\hline
\end{tabular}

Sd: Standard deviation.

$\mathrm{t}$ : Independent $\mathrm{t}$ test MW: Mann Whitney test

NS: Non significant $(\mathrm{p}>0.05) \quad$ *: Significant $(\mathrm{P}<0.05) \quad * *$ : Highly significant $(\mathrm{p}<0.01)$

Table (6): Comparison between the two studied groups in sexual function index score after treatment.

\begin{tabular}{|c|c|c|c|c|c|}
\hline \multicolumn{2}{|c|}{ Variable } & $\begin{array}{c}\text { Group I (Vardenafil) } \\
(\mathbf{n}=16)\end{array}$ & $\begin{array}{c}\text { Group II (Flibanserin) } \\
(\mathbf{n = 1 6 )}\end{array}$ & Test & $\mathbf{P}$ \\
\hline Desire & $\begin{array}{l}\text { Mean } \pm \text { Sd } \\
\text { Median (Range) }\end{array}$ & $\begin{array}{l}5.44 \pm 1.55 \\
6.5(3-9)\end{array}$ & $\begin{array}{l}6.45 \pm 1.17 \\
5.5(4-10)\end{array}$ & $\begin{array}{c}\mathrm{t} \\
2.08\end{array}$ & $0.04 *$ \\
\hline Arousal & $\begin{array}{l}\text { Mean } \pm \text { Sd } \\
\text { Median (Range) }\end{array}$ & $\begin{array}{l}2.44 \pm 0.73 \\
3(2-4)\end{array}$ & $\begin{array}{c}2.88 \pm 0.89 \\
2(2-4)\end{array}$ & $\begin{array}{c}\mathrm{t} \\
1.53\end{array}$ & $\begin{array}{l}0.14 \\
\mathrm{NS}\end{array}$ \\
\hline Lubrication & $\begin{array}{l}\text { Mean } \pm \text { Sd } \\
\text { Median (Range) }\end{array}$ & $\begin{array}{c}3.19 \pm 0.54 \\
2(2-4)\end{array}$ & $\begin{array}{l}3.19 \pm 0.91 \\
1.5(2-4)\end{array}$ & $\begin{array}{c}\mathrm{t} \\
00\end{array}$ & $\begin{array}{l}1.00 \\
\mathrm{NS}\end{array}$ \\
\hline Orgasm & $\begin{array}{l}\text { Mean } \pm \text { Sd } \\
\text { Median (Range) }\end{array}$ & $\begin{array}{l}2.06 \pm 0.77 \\
3(1-3)\end{array}$ & $\begin{array}{l}2.69 \pm 0.79 \\
2.5(2-4)\end{array}$ & $\begin{array}{l}\text { MW } \\
2.26\end{array}$ & $\mathbf{0 . 0 3}^{*}$ \\
\hline Satisfaction & $\begin{array}{l}\text { Mean } \pm \text { Sd } \\
\text { Median (Range) }\end{array}$ & $\begin{array}{l}3.44 \pm 0.89 \\
3(2-5)\end{array}$ & $\begin{array}{l}3.63 \pm 0.72 \\
2(2-5)\end{array}$ & $\begin{array}{l}\mathrm{t} \\
.66\end{array}$ & $\begin{array}{l}0.52 \\
\mathrm{NS}\end{array}$ \\
\hline Pain & $\begin{array}{l}\text { Mean } \pm \text { Sd } \\
\text { Median (Range) }\end{array}$ & $\begin{array}{l}0.5 \pm 0.52 \\
0.5(0-1)\end{array}$ & $\begin{array}{c}0.56 \pm 0.51 \\
0(0-1)\end{array}$ & $\begin{array}{l}\text { MW } \\
-.35\end{array}$ & $\begin{array}{l}0.73 \\
\mathrm{NS}\end{array}$ \\
\hline Full score & $\begin{array}{l}\text { Mean } \pm \text { Sd } \\
\text { Median (Range) }\end{array}$ & $\begin{array}{l}17.1 \pm 2.46 \\
17(13-21)\end{array}$ & $\begin{array}{c}19.37 \pm 3.81 \\
14.5(13-25)\end{array}$ & $\begin{array}{c}\mathrm{t} \\
2.1\end{array}$ & $0.04 *$ \\
\hline
\end{tabular}

Sd: Standard deviation.

NS: Non significant $(\mathrm{p}>0.05)$

DISCUSSION

The present study showed that there were no statistical significant differences between the studied $\mathrm{t}$ : Independent $\mathrm{t}$ test

*: Significant $(\mathrm{P}<0.05)$
MW: Mann Whitney test

$* *$ : Highly significant $(\mathrm{p}<0.01)$

groups in any of demographic data. This is supported by study of Basson $\boldsymbol{e t}$ al. ${ }^{(8)}$ as they reported that there was no statistically significant difference between both 
groups regarding demographic data. While, this is in contrast with study of Basson and Brotto ${ }^{(9)}$ as they found that the mean age of the women was $56.6 \pm 6.6$ years (range: $40-78$ years) with a mean educational attainment of $15.0 \pm 2.9$ years (range: 9-21 years).

In the study in our hands, there were no statistical significant differences between the two studied groups in all scores pretreatment. However, there was statistical significant increase in desire, orgasm and total score among group II (flibanserin) compared to group I (vardenafil) post-treatment.

Regarding comparing pre- and post- treatment scores in each group: In Group I, there were statistical significant increase in index scores of lubrication and satisfaction $(37.5 \%$ \& $42.7 \%$ respectively) posttreatment. In addition, there was statistical significant increase in orgasm and total score $(25 \%$ \& $23.36 \%$ respectively) post-treatment compared to pretreatment. While in group II, there was highly statistical significant increase in desire, orgasm, satisfaction and total $(33.3 \%, 41.76 \% \& 25.66 \%$ respectively) posttreatment. Moreover, there was statistical significant decrease in arousal score $(23.75 \%)$ post treatment compared to pretreatment. Our results are supported by study of Robinson et $\boldsymbol{a l} .{ }^{(10)}$ as they reported that flibanserin is effective in the treatment of HSDD. Flibanserin should be administered at bedtime to limit the risk for hypotension/syncope, accidental injury and central nervous system (CNS) depression. Concomitant alcohol use contributes to significant CNS depression and hypotension/syncope with flibanserin and should be avoided according to the boxed warning. Careful patient assessment prior to the diagnosis of HSDD and the use of flibanserin is needed for safe use. Katz et al. (11) in their study of efficacy of flibanserin in women with HSDD from the BEGONIA trial found that flibanserin $100 \mathrm{mg}$ qhs resulted in significant improvements in the number of SSE and sexual desire (FSFI desire domain score) vs. placebo. Flibanserin was associated with significant reductions in distress associated with sexual dysfunction (FSDS-R total score) and distress associated with low sexual desire (FSDS-R Item 13) vs placebo. DeRogatis et al. ${ }^{(12)}$ studied the efficacy of 24 weeks' flibanserin 50 and 100 $\mathrm{g}$ treatment in premenopausal women with HSDD Violet study. At the end of the study, mean (SE) increase from baseline in FSFI desire domain score was $0.5 \pm 0.1$ for placebo, $0.8 \pm 0.1$ for flibanserin $50 \mathrm{mg}(P$ $<0.05$ vs. placebo), and $0.9 \pm 0.1$ for flibanserin 100 $\mathrm{mg}(P<0.000,1$ vs. placebo $)$. The greater increases in FSFI desire domain score in both flibanserin groups vs. placebo were statistically significant at all-time points $(P<0.05$ vs. placebo for all), except at week 4 for flibanserin $50 \mathrm{mg}$. Frühauf et al. ${ }^{(13)}$ suggested that the benefits of flibanserin treatment are marginal, particularly when taking into account the concurrent occurrence of AEs. It has been suggested that women with HSDD would benefit most from an integrative approach, including, medical, psychiatric, psychological, couple-relationship and sociocultural domains: the biopsychosocial model. Before flibanserin can be recommended in guidelines and clinical practice, future studies should include women from diverse populations, particularly women with a history of somatic and psychological comorbidities, medication use and surgical menopause.

Caruso et al. ${ }^{(14)}$ examined the effects of 100 $\mathrm{mg}$ sildenafil on sexual functioning in 32 premenopausal women with female sexual arousal disorder (FSAD) and type I diabetes using a double blind, crossover and placebo -controlled design over two 8-weeks periods. Doppler ultrasonography was used to measure clitoral artery blood flow during placebo and sildenafil treatment. Significant increases in sexual arousal and orgasm, and reduction in dyspareunia were reported for the sildenafil versus placebo conditions (assessed using the PEQ), and significant increases in clitoral blood flow were reported for the sildenafil condition.

DasGupta and Fowler ${ }^{(15)}$ examined the efficacy of sildenafil in women with sexual dysfunction related to a diagnosis of multiple sclerosis using a double blind, randomized, placebo-controlled and crossover design with an open label extension phase. Nineteen women reported sexual dysfunction, primarily concerns with lubrication, sensation, and orgasm based on pretreatment assessment using the SFQ. They were randomized to either $50 \mathrm{mg}$ sildenafil or placebo. At 2 weeks, women's sildenafil dosage was modified in response to negative side effects (reduced to $25 \mathrm{mg}$ ) or lack of treatment response (increased to $100 \mathrm{mg}$ ). Sildenafil use resulted in significant improvement in lubrication and genital sensation (as assessed using the SFQ) during the double blind phase (compared to baseline, not placebo) and significantly improved orgasm in an open label phase. Furthermore, Nurnberg et al. (16) used an 8-week prospective, parallel group, randomized, double blind, placebocontrolled clinical trial to determine if sildenafil improved sexual functioning among women with serotonin reuptake inhibitor-associated sexual dysfunction. Outcomes were defined as change in the Clinical Global Impression scale (CGI), Sexual Functioning Questionnaire (SFQ), Arizona Sexual Experience Scale, and the University of New Mexico Sexual Function Inventory at weeks 2, 4, and 8. Symptom reduction was noted on the CGI with sildenafil treatment. Moreover, orgasm subscales of all inventories (ability to achieve a satisfaction with) showed improvement with sildenafil use.

Finally, comprehensive assessment of the role of physiological and subjective factors in women with FSAD by the clinician may be helpful in determining which women are more likely to respond to pharmacological and psychological treatment. Women with genital arousal disorder may respond to PDE5. Unlike women with subjective or combined subjectivegenital arousal disorder, particularly as deficits in 
genital response, measured using vaginal photoplethysmography, which have been observed among women with the genital arousal subtype of FSAD. Future research using careful classification of subtypes of FSAD and psychophysiological assessment of genital subjective concordance will help clarify both the role of genital response in women's sexual pleasure, and the role of PDE5 in the treatment of FSAD ${ }^{(17)}$.

\section{CONCLUSION}

Treatment of FSD is multi-factorial. Medications alone do not resolve FSD. Flibanserin is a controversial drug approved for a controversial disorder amid huge controversy. While, it may serve as the lamp in the long search for female sexual problems, it has still a long way to go. Women taking this drug must well be educated about the adverse events associated with this drug and the possible interactions. Flibanserin-treated women reported improvements on most measures of sexual dysfunction during the study. Trend was observed on most study measures in favor of flibanserin and significant differences were noted to compare with vardenafil.

\section{REFERENCES}

1. Salonia A, Zanni G, Nappi RE et al. (2004): Sexual dysfunction is common in women with lower urinary tract symptoms and urinary incontinence: results of a cross-sectional study. Eur Urol., 45: 642-8.

2. Allahdadi K, Tostes R, Webb R (2008): Female Sexual Dysfunction: Therapeutic options and experimental challenges. Journal of Sexual Medicine, 7 (4): 260-269.

3. Marazziti D, Palego L, Giromella A et al. (2002): Regiondependent effects of flibanserin and buspirone on adenylyl cyclase activity in the human brain. Int. J. Neuropsychopharmacol., 5 (2): 131-140.

4. Allers K, Dremencov E, Ceci A et al. (2010): Acute and repeated flibanserin administration in female rats modulates monoamines differentially across brain areas: a microdialysis study. J Sex Med., 7: 1757-1767.

5. Gragasin F, Michelakis E, Hogan A et al. (2004): The neurovascular mechanism of clitoral erection: nitric oxide and cGMP-stimulated activation of $\mathrm{BKCa}$ channels. FASEB J., 18 (12): 1382-1391.
6. Corbin J (2004): Mechanisms of action of PDE5 inhibition in erectile dysfunction. Int J Impot Res., 16 (1): 4-8.

7. Kandil A, Galal E, Shawqy A (2017): Evaluation of the sildenafil effect on female sexual dysfunction. Human Andrology, 3: 84-91.

8. Basson R, McInnes R, Smith M et al. (2002): Efficacy and safety of sildenafil citrate in women with sexual dysfunction associated with female sexual arousal disorder. J Womens HealthmGend Based Med., 11:36777.

9. Basson R, Brotto L (2003): Sexual psychophysiology and effects of sildenafil citrate in oestrogenised women with acquired genital arousal disorder and impaired orgasm: a randomised controlled trial. BJOG: An International Journal of Obstetrics and Gynaecology, 110 (11): 1014-1024.

10. Robinson K, Cutler J, Carris N (2016): First pharmacological therapy for hypoactive sexual desire disorder in premenopausal women: Flibanserin. Ann Pharmacother., 50: 125-32. 20.

11. Katz M, DeRogatis L, Ackerman $R$ et al. (2013): Efficacy of flibanserin in women with hypoactive sexual desire disorder: Results from the BEGONIA trial. J Sex Med., 10: 1807-1815.

12. DeRogatis L, Molly K, Michèle $M$ et al. (2012): Efficacy of Flibanserin in Women with HSDD. J Sex Med., 9: 1074-1085.

13. Frühauf S, Gerger H, Schmidt H et al. (2013): Efficacy of psychological interventions for sexual dysfunction: a systematic review and meta-analysis. Arch Sex Behav., 42 (6): 915-933.

14. Caruso S, Rugolo S, Agnello C et al. (2008): Sildenafil improves sexual functioning in premenopausal womenwith type 1 diabetes who are affected by sexual arousal disorder: A double blind, crossover, placebo-controlled pilot study. Fertil Steril., 85: 1496-501.

15. DasGupta R, Fowler C (2004): Bladder, Bowel and Sexual Dysfunction in Multiple Sclerosis: Management Strategies. Drugs, 63: 153-166.

16. Nurnberg H, Hensley $P$, Heiman $J$ et al. (2008): Sildenafil treatment of women with antidepressantassociated sexual dysfunction: a randomized controlled trial. JAMA., 300: 395-404.

Brotto L, Basson R, Gorzalka B (2004): Psychophysiological assessment in premenopausal sexual arousal disorder. J Sex Med., 1: 266-77. 